Research Article

\title{
Dispersion Compensation Method for Lamb Waves Based on Measured Wavenumber
}

\author{
J. Li $\mathbb{D}^{1,2}$ and Y. Han $\mathbb{D}^{1}$ \\ ${ }^{1}$ Shanxi Key Laboratory of Signal Capturing \& Processing, North University of China, Taiyuan 030051, China \\ ${ }^{2}$ Lvliang University, Lvliang 033000, China \\ Correspondence should be addressed to Y. Han; hanyan@nuc.edu.cn
}

Received 5 September 2019; Revised 10 January 2020; Accepted 13 January 2020; Published 22 February 2020

Academic Editor: Jean-Mathieu Mencik

Copyright (c) $2020 \mathrm{~J}$. Li and Y. Han. This is an open access article distributed under the Creative Commons Attribution License, which permits unrestricted use, distribution, and reproduction in any medium, provided the original work is properly cited.

In this study, a delta wavenumber dispersion compensation ( $\triangle \mathrm{K}-\mathrm{DC}$ ) method was developed and applied, not only with the theoretical wavenumber but also with the measured wavenumber. Dispersion compensation can be achieved by the following steps: relative wavenumber measurement, traveling distance estimation, phase compensation, and wave correction. The feasibility of $\triangle \mathrm{K}$-DC with the theoretical wavenumber and measured wavenumber was validated with a high-dispersive $\mathrm{A}_{0}$ mode in a $2 \mathrm{~mm}$ steel plate experiment. The results showed that phase spectrum measurement was an effective method to construct the wavenumber curve, the propagation distances estimated by SAP ${ }^{2}$ were very accurate, and the dispersive signals can be compensated perfectly by applying the phase compensation and wave correction methods for each wavepacket. The present results highlight the application of $\triangle \mathrm{K}-\mathrm{DC}$ on dispersion compensation without any material parameters of a waveguide.

\section{Introduction}

Ultrasonic-guided wave is an efficient approach to nondestructive testing (NDT) [1-4] and structural health monitoring (SHM) $[5,6]$ and material characterization applications [7]. Lamb waves are commonly used in plate structures because of the possibility of long traveling distance, large detecting area, and excellent inspecting sensitivities. Dispersion and multimode are fundamental properties of Lamb waves. Dispersion characteristics are that velocity depends on the product of excitation frequency and plate thickness and even relates to the layup orientation for anisotropic materials. Multimode property is that a finite waveguide can support infinite modes. A nonlinear relation between wavenumber and frequency results in wavepacket extension and waveform distortion when the Lamb wave propagates through a plate structure. The longer the propagation time is, the more serious the dispersion phenomenon is. In addition, considering the fact that the acquiring signal is the accumulation of multiple modes from the excitation point, scattered waves and new converted modes from the damage, and reflected waves from the boundary, overlapping of these waveforms makes signals difficult to interpret accurate damage identification and high-resolution imaging hard to develop. Therefore, it is of great significance to explore the dispersion compensation method for developing efficient NDT methods.

More recently, there have been a number of techniques proposed for dispersion compensation or dispersion removal, such as the optimal design of the excitation waveform, signal domain transform, linearization mapping, warped frequency transform, and time reversal. Usually, a narrowband excitation signal can largely reduce the dispersion phenomenon in the frequency domain, but it makes signals more likely to overlap in the time domain. Signal domain transform is a widely used method to make waves free from dispersion and waveform deformation. Cai [8] and Wilcox [9] proposed a time-distance mapping method. Firstly, a dispersive Lamb wave is mapped from the time domain to the frequency domain using fast Fourier transform (FFT). Secondly, it is interpolated to the wavenumber domain based on the prior wavenumber knowledge, and finally, the signal is transformed to the distance domain using inverse fast Fourier transform (IFFT). Cai [10, 11] 
developed a linear mapping technique. This method was realized based on the fact that the nonlinear relation between wavenumber and frequency leads to wave dispersion. Firstly, a linear wavenumber was constructed by expanding the nonlinear wavenumber in a Taylor series around the central frequency up to the first order. Then, in the wavenumber domain, the nonlinear wavenumber was mapped by the linear one to remove the dispersion effect. Backpropagation was also a signal processing tool of effective compensation, such as the time-reversal technique [12-15]. The dispersive signal was reversed in the time domain and reemitted from the sensor location to the actuator location. Fu [16] and Marchi $[17,18]$ presented the warped frequency transform (WFT), which maps signals from the frequency domain into the warped frequency domain by a warping function. Except for these commonly used compensation methods, Sicard [19] established a scalar diffraction theory, a numerical dispersion compensation technique with time recompression. Hua [20] performed the dispersion compensation by applying time reversed on the excitation signal with a given distance. $\mathrm{Xu}$ [21] developed a dispersion compensation method based on compressed sensing.

Together, most studies highlighted the needs for the prior knowledge on the dispersion characteristics (phase velocity, group velocity, or wavenumber) and the applications of FFT, IFFT, and interpolation operation. Time reversal does not need any prior dispersion knowledge nor material properties. The present study is to develop the $\Delta \mathrm{K}$ DC method, not only applied for the theoretical wavenumber but also applied for the measured wavenumber. When the theoretical wavenumbers are available, dispersion compensation can be approached by phase compensation and wave correction. When the structure properties are unknown, dispersion compensation can be achieved by the following steps: wavenumber measurement, traveling distance estimation, phase compensation, and wave correction. The phase spectrum measurement method is developed to measure the relative wavenumbers. The wavelet energy spectrum method is provided to extract the flight time of each wavepacket. The propagating distances are estimated by the group velocity and the flight times; finally, phase compensation and wave correction are executed to compensate dispersion separately. Stimulation and experimental setup are then performed to validate the performances of $\triangle \mathrm{K}$-DC for the theoretical wavenumber and the measured wavenumber.

\section{Fundamental Investigation on $\triangle \mathrm{K}$-DC with Theoretical Wavenumber}

A nonlinear relation between wavenumber and frequency results in dispersion effect, which can be minimized by compensating a nonlinear wavenumber to the corresponding linear one. The dispersive signals that are satisfactorily renovated to the waveform of the activation signal are the purpose of $\triangle \mathrm{K}$-DC.
2.1. The Basic Principle of $\triangle K-D C$. Lamb waves can be modeled by imposing traction-free surface boundary conditions on the equations of motion, effectively describing the wave behavior. From Rose [22], the propagation of guided waves can be obtained from the dispersion characteristics of a plate structure and the spectrum of an excitation signal. Dispersive waves can be generated using the following equations:

$$
\begin{aligned}
& Y(r, \omega)=Y(0, \omega) H(r, \omega), \\
& H(r, \omega)=A(r, \omega) e^{-i K(\omega) r},
\end{aligned}
$$

where $r$ is the propagation distance; $\omega$ is the angular frequency and $\omega_{c}$ is the angular frequency at the central frequency of the excitation signal; $Y(0, \omega)$ and $Y(r, \omega)$ are the spectrums of the excitation signal $y(0, t)$ and Lamb wave $y(r, t)$; and $H(r, \omega)$ is the transfer function, where $K(\omega)$ denotes the nonlinear wavenumber. The slightly varied amplitude $A(r, \omega)$ can be simplified as "1" to facilitate the following analysis. Based on the time-shifting properties of FFT, equation (1b) indicates that the complex phase shifts of $Y(0, \omega)$ in the frequency domain, $e^{-i K(\omega) r}$, are interpreted as a translation of $y(0, t)$ in the time domain, $K(\omega) r / \omega$.

According to the dispersion property and energy conservation, different frequency components in a wave packet will propagate at different velocities, which may result in the broadening of wavepackets and reduction of amplitude when they propagate through a structure. As discussed above, both dispersion phenomenon and waveform distortion of $y(r, t)$ can be achieved by linearizing the nonlinear wavenumber. The linear wavenumber, $K_{\text {lin }}(\omega)$, can be obtained by expanding $K(\omega)$ as the Taylor series around the central frequency up to the first order, as shown in equation (2). Equation (3) shows the 0-order coefficient is the wavenumber at $\omega_{c}$ and the 1-order coefficient is the reciprocal of group velocity at $\omega_{c}$, where $c_{p}\left(\omega_{c}\right)$ and $c_{g}\left(\omega_{c}\right)$ express the phase velocity and the group velocity at $\omega_{c}$, respectively. The delta wavenumber, $\Delta K(\omega)$, is calculated by equation (4).

$$
K_{\operatorname{lin}}(\omega)=K\left(\omega_{c}\right)+\left.\left(\frac{\mathrm{d} K(\omega)}{\mathrm{d} \omega}\right)\right|_{\omega=\omega_{c}}\left(\omega-\omega_{c}\right)=k_{0}+k_{1}\left(\omega-\omega_{c}\right),
$$

$$
\begin{aligned}
& k_{0}=\frac{\omega_{c}}{c_{p}\left(\omega_{c}\right)}, \\
& k_{1}=\frac{1}{c_{g}\left(\omega_{c}\right)},
\end{aligned}
$$

$\Delta K(\omega)=K_{\operatorname{lin}}(\omega)-K(\omega)$

Substituting equations (2) and (3) into equation (1), the nondispersive signal, $Y_{\operatorname{lin}}^{\prime}(r, \omega)$, is obtained by using the linear wavenumber as 


$$
\begin{gathered}
Y_{\operatorname{lin}}^{\prime}(r, \omega)=Y(0, \omega) e^{-i K_{\operatorname{lin}}(\omega) r}=Y(0, \omega) e^{i \varphi_{0} r} e^{-i\left(\omega r / c_{g}\left(\omega_{c}\right)\right)}, \\
\varphi_{0}=\omega_{c}\left(\frac{1}{c_{g}\left(\omega_{c}\right)}-\frac{1}{c_{p}\left(\omega_{c}\right)}\right) . \\
y_{\text {lin }}^{\prime}(r, t) \text { is obtained by applying IFFT to } Y_{\operatorname{lin}}^{\prime}(r, \omega) \text { as } \\
y_{\text {lin }}^{\prime}(r, t)=\operatorname{IFFT}\left(Y_{\operatorname{lin}}^{\prime}(r, \omega)\right)=y\left(0, \frac{t-r}{c_{g}\left(\omega_{c}\right)}\right) e^{i \varphi_{0} r}, \\
y_{\operatorname{lin}}(r, t)=y\left(0, \frac{t-r}{c_{g}\left(\omega_{c}\right)}\right)=y_{\operatorname{lin}}^{\prime}(r, t) e^{-i \varphi_{0} r} .
\end{gathered}
$$

Equation (7) indicates $y_{\text {lin }}^{\prime}(r, t)$ is an entire $r / c_{g}\left(\omega_{c}\right)$ delay of $y(0, t)$ in the time domain, with another phase factor $\varphi_{0} . y_{\text {lin }}(r, t)$ is only an entire $r / c_{g}\left(\omega_{c}\right)$ delay of $y(0, t)$ in the time domain based on the fact that a constant time delay does not result in dispersion, as illustrated in equation (8). Usually, $c_{g}\left(\omega_{c}\right) \neq c_{p}\left(\omega_{c}\right), \exp \left(-i \varphi_{0}\right) \neq 1$, so the waveform of $y_{\text {lin }}^{\prime}(r, t)$ may differ from that of $y(r, t)$. By applying equations (1), (5), and (8), the relationship between $Y_{\operatorname{lin}}(r, \omega)$ and $Y(r, \omega)$ can be described in equation (9), with $Y_{\text {lin }}^{\prime}(r, t)$ as the intermediate function. $y_{\operatorname{lin}}(r, t)$ can be obtained by applying IFFT to $Y_{\text {lin }}(r, \omega)$, in equation (10).

$$
\begin{gathered}
Y_{\operatorname{lin}}(r, \omega)=Y_{\operatorname{lin}}^{\prime}(r, \omega) e^{-i \varphi_{0} r}=Y(r, \omega) e^{-i \Delta K(\omega) r} e^{-i \varphi_{0} r}, \\
y_{\operatorname{lin}}(r, t)=\operatorname{IFFT}\left(Y_{\operatorname{lin}}(r, \omega)\right) .
\end{gathered}
$$

To sum up, phase compensation and waveform correction are the specific steps for the compensation process, as indicated in equation (9).

\subsection{Realization of $\triangle K-D C$ with Theoretical Wavenumber.} The procedure of $\triangle \mathrm{K}-\mathrm{DC}$ with the known wavenumber and distance is given in detail, as shown in Figure 1. Compared with the traditional dispersion compensation algorithm, interpolation and transforming in the wavenumber domain are not required. The approach includes the following steps sequentially:

(a) Obtaining $K(\omega)$ by solving the dispersion equation, constructing $K_{\operatorname{lin}}(\omega)$ with equation (2), and getting $\Delta K(\omega)$ with equation (4), respectively

(b) Applying N-point FFT to $y(r, t)$ for $Y(r, \omega)$

(c) $Y_{\operatorname{lin}}^{\prime}(r, t)$ is compensated by the phase-delay factor $e^{-i \Delta K(\omega) r}$ to $Y(r, \omega)$ with equation (9)

(d) Correcting the waveform with $e^{-i \varphi_{0} r}$ to get $Y_{\operatorname{lin}}(r, \omega)$

(e) Applying N-point IFFT to $Y_{\text {lin }}(r, \omega)$ for $y_{\operatorname{lin}}(r, t)$

2.3. Simulation on $\triangle K-D C$ in a Steel Plate. To validate the feasibility of the $\triangle \mathrm{K}-\mathrm{DC}$ algorithm with the theoretical wavenumber, a single $A_{0}$ mode wave was activated in a homogeneous and isotropic steel plate (elastic modulus: $208.42 \mathrm{GPa}$, density: $7850 \mathrm{~kg} / \mathrm{m}^{3}$, Poisson's ratio: 0.2959 , and thickness: $2 \mathrm{~mm}$ ). In a pitch-catch configuration, 3.5-cycle

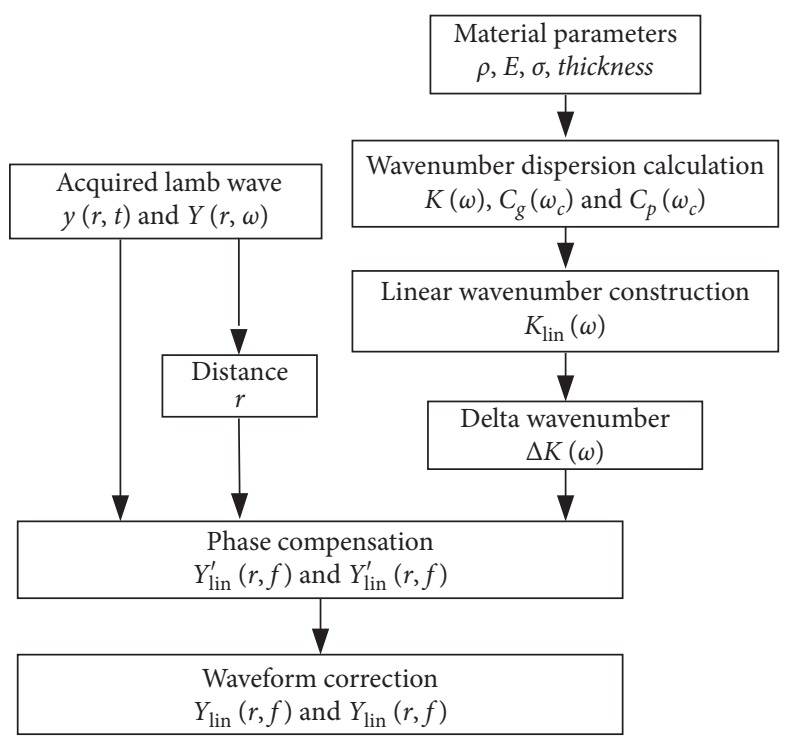

FIGURE 1: Schematic of $\Delta \mathrm{K}-\mathrm{DC}$ for the theoretical wavenumber.

sinusoid tone bursts at a central frequency of $200 \mathrm{kHz}$ modulated by a Hanning window were applied in turn to activate a single $\mathrm{A}_{0}$ mode. And the sensor signal was received upon traveling $300 \mathrm{~mm}$ across the plate. The FEM simulation was accomplished using ABAQUS ${ }^{\circledast} /$ EXPLICIT, as shown in Figure 2(a). The sampling frequency was $10 \mathrm{MHz}$. Displacement gram in the thickness direction is illustrated in Figure 2(b). It can be seen that the wavepacket spreads out in space and time and amplitude reduces when Lamb waves propagate through the plate.

It can be seen that the time duration rises from $17.5 \mu$ s of the excitation signal (shown in Figure 3(a)) to about $50 \mu$ s of the captured signal (shown in Figure 3(b)). The theoretical velocity dispersion curves can be obtained by solving the dispersion equation (shown in Figure 3(c)). The group velocity and the phase velocity at $200 \mathrm{kHz}$ are $2794.4 \mathrm{~m} / \mathrm{s}$ and $1724.8 \mathrm{~m} / \mathrm{s}$, respectively. Then, the nonlinear wavenumber $K(\omega)$, the linear wavenumber $K_{\operatorname{lin}}(\omega)$, and the delta wavenumber $\Delta K(\omega)$ were successfully calculated with equations (2)-(4) (shown in Figure 3(d)). As illustrated in Figure 3(e), the phase compensation result of $y(r, t)$, $y_{\text {lin }}^{\prime}(r, t)$, was still different from the waveform of $y(0, t)$ mainly because of the existence of $\varphi_{0}$. Following this, it was necessary to correct the waveform as equation (10), and what stands out in Figure 3(f) was perfect compensation for the dispersive signal.

\section{3. $\triangle \mathrm{K}$-DC with the Measured Wavenumber}

When the material parameters are unavailable, such as elastic modulus, density, and Poisson's ratio for the isotropy structure and stiffness matrix and layup for anisotropic structures, the wavenumber could not be theoretically computed. In this event, dispersion compensation can be achieved by the following procedures: wavenumber measurement, traveling distance estimation, phase compensation, and wave correction. The feasibility of the scheme was verified in a $2 \mathrm{~mm}$ steel plate. 


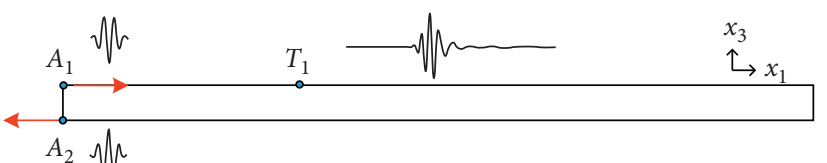

(a) (b)

Figure 2: Simulation setup in a steel plate. (a) ABAQUS model. (b) Displacement gram in the thickness direction.

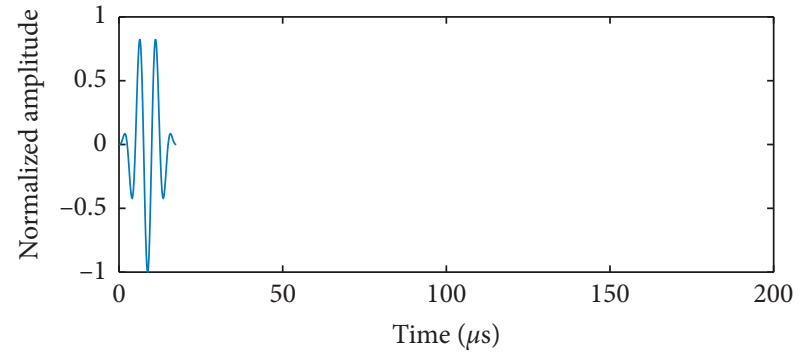

_ Excitation signal

(a)

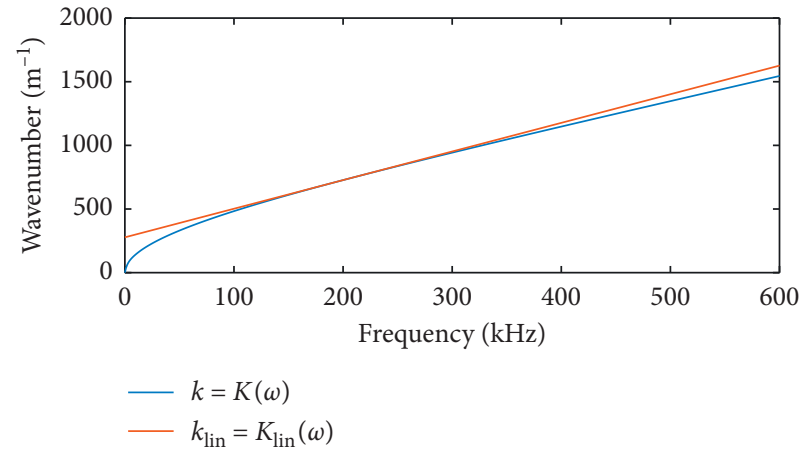

(c)

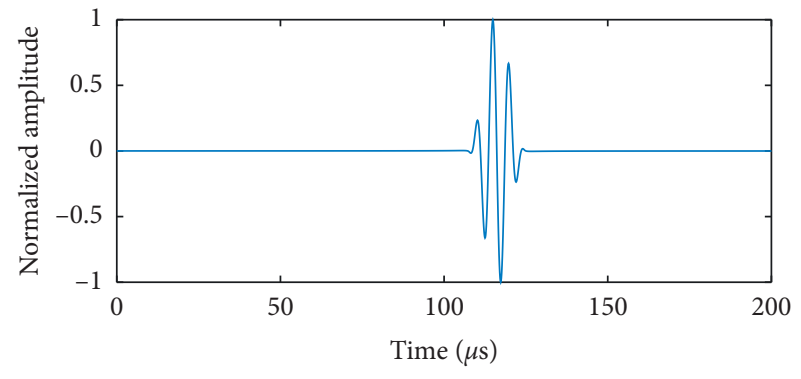

_ Phase compensation result

(e)

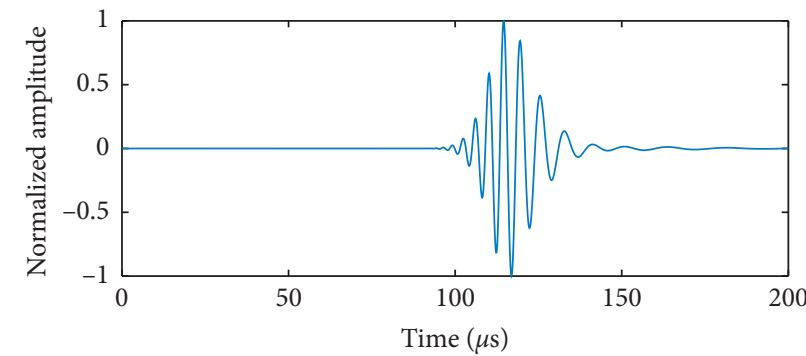

- $\mathrm{A}_{0}$ wave

(b)

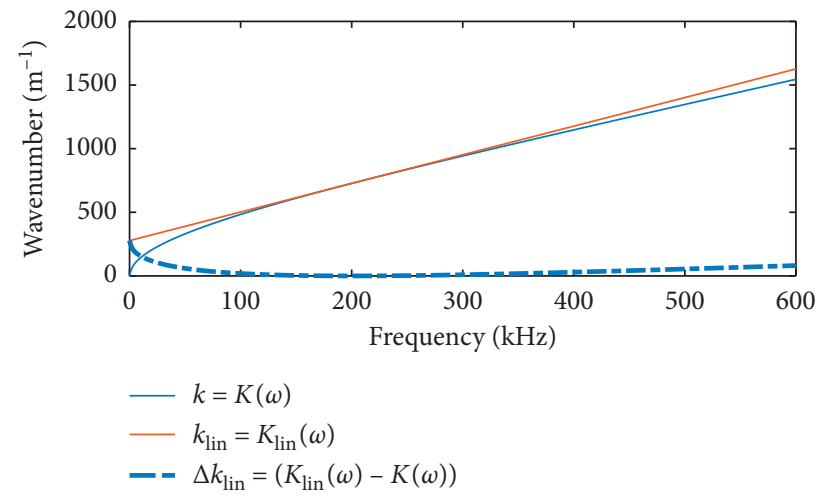

(d)

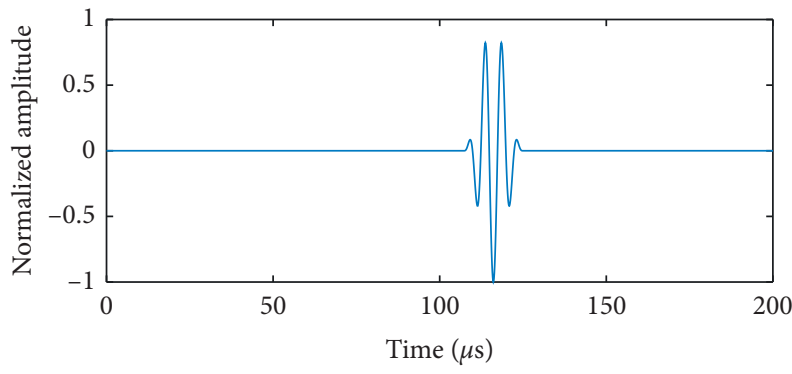

_ Waveform correction

(f)

Figure 3: Procedure of $\triangle \mathrm{K}$-DC with the known wavenumber: (a) excitation signal in the time domain $y(0, t)$; (b) sensor signal in the time domain $y(r, t)$; (c) velocity dispersion curves $c_{p}(\omega)$ and $c_{g}(\omega)$; (d) wavenumber curves $K(\omega), K_{\text {lin }}(\omega)$, and $\Delta K(\omega)$; (e) phase compensation result $y_{\text {lin }}^{\prime}(r, t)$; (f) waveform correction result $y_{\text {lin }}(r, t)$.

\subsection{Phase Spectrum Method of Wavenumber Measured. A} phase spectrum measurement method is proposed to measure the wavenumber based on the phase difference between the excitation signal and the Lamb wave, expressed as equations (11)-(13). This process needs the spectrum of the excitation signal and Lamb waves but does not need any structure material parameters.

$$
\begin{aligned}
& H(r, \omega)=\frac{Y(r, \omega)}{Y(0, \omega)}=e^{-i \Delta \Phi(\omega)}=e^{-i K(\omega) r}, \\
& \Delta \Phi(\omega)=\text { unwrap }\left(\arctan \left[\frac{\operatorname{Im}}{\operatorname{Re}}\right]\right)
\end{aligned}
$$




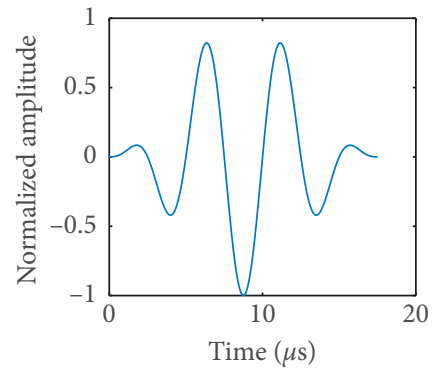

(a)

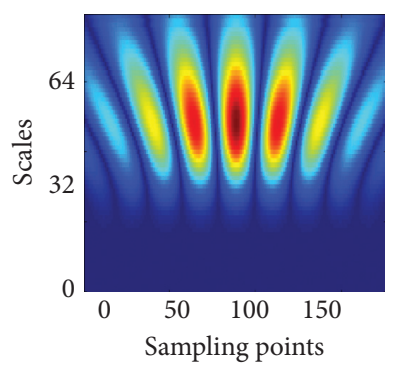

(b)

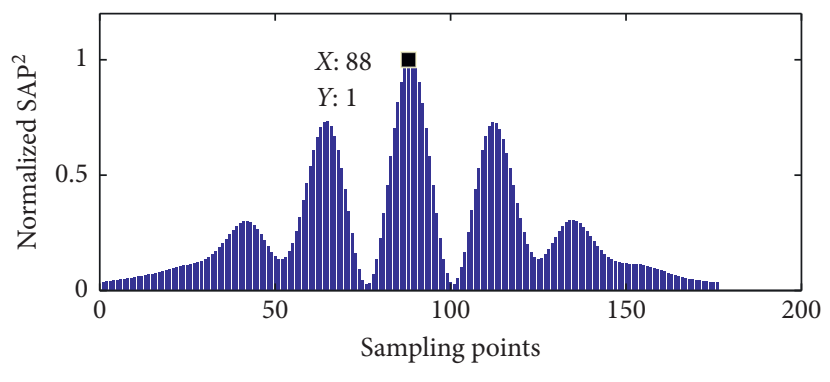

Excitation signal

FIgURE 4: Procedure of $\mathrm{SAP}^{2}$ : (a) excitation signal; (b) wavelet spectrum of (a); (c) $\mathrm{SAP}^{2}$ of (a).

$$
K(\omega)=\frac{\Delta \Phi(\omega)}{r}
$$

where $\Delta \Phi(\omega)$, the unwrapped phase of $H(r, \omega)$, can be calculated from the arctangent function in equation (12), where Re and Im represent the real part and the imaginary part of $H(r, \omega)$, respectively. Equation (13) presents that the dispersion number is the ratio of phase difference to propagation distance. Therefore, the propagation distance $r$ for measuring the wavenumber should be accurately known, or the measured wavenumber is not accurate.

\subsection{Wavelet Energy Spectrum for Traveling Distance}

Extraction. The aim of distance estimation is to determine whether the measured group velocity is accurate and use the estimated distances to compensate dispersion. Extracting the exact arrival time is a critical step to determine the propagation distance. The scale-averaged wavelet power $\left(\mathrm{SAP}^{2}\right)$ based on CWT, which is defined in equations (14) and (15) $[4,23]$, is used to determine the exact energy peak of $y(r, t)$, where $M$ is the largest scale and $N$ is the sampling point of $y$ $(r, t)$. The mean wave energy, $E$, over the entire time period is expressed in equation (15). Figure 4(b) displays $\mathrm{SAP}^{2}$ of the excitation signal.

$$
\begin{aligned}
\operatorname{SAP}^{2}(n) & =\frac{1}{M} \sum_{i=1}^{M}\left|\operatorname{CWT}\left(a_{i}, n\right)\right|^{2}, \quad i=1,2, \ldots, M, \\
E & =\frac{1}{N} \sum_{i=1}^{M} \operatorname{SAP}^{2}(n), \quad n=1,2, \ldots, N, \\
\text { time }_{\text {flight }} & =\text { time }_{\text {arrival }}(y(r, t))-\text { time }_{\text {arrival }}(y(0, t)), \\
r & =c_{g}\left(\omega_{c}\right) \cdot \text { time }_{\text {flight }} .
\end{aligned}
$$

According to Rose [22], wave propagation is energy transportation, and the group velocity is the velocity of energy transportation. The flight time of each wavepacket should be defined in terms of the time difference between the arrival time and the initial time moment, as expressed in equation (16). The arrival times of multiple wavepackets of $y$ $(r, t)$ are determined at the moments that the energy reached

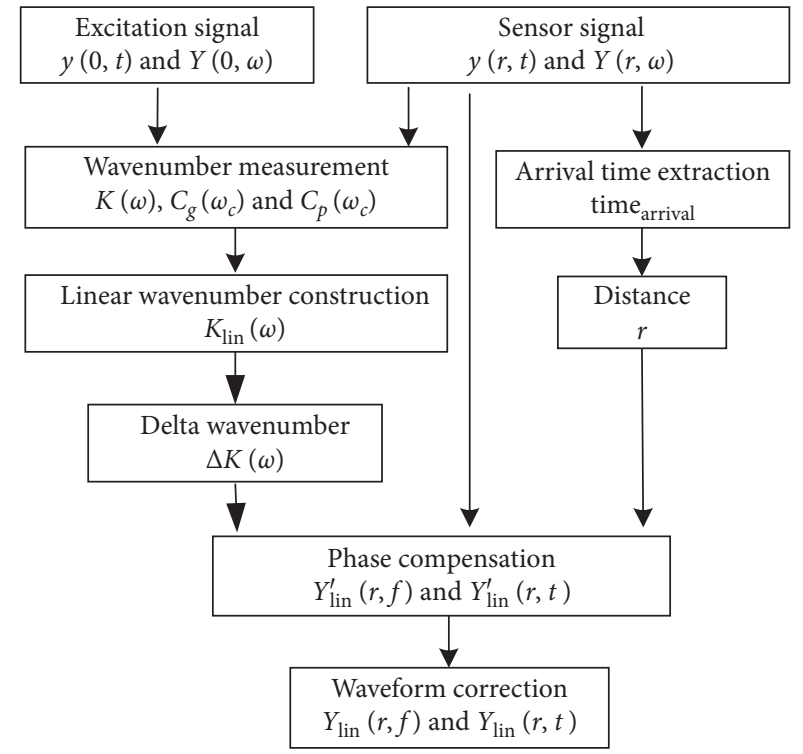

FIGURE 5: Schematic of $\triangle \mathrm{K}-\mathrm{DC}$ for the measured wavenumber.

its extremums in the time-scale domain. Looking at Figure 4(c), it is apparent that the initial time of the excitation signal is the 88th sampling point. Propagating distances are estimated by the group velocity at the central frequency and the flight time of each wavepacket, as expressed in equation (17).

3.3. Realization of $\triangle K-D C$ with Measured Wavenumber. As discussed above, the procedures of the $\triangle \mathrm{K}$-DC algorithm can be summarized, as displayed in Figure 5. The approach includes the following steps sequentially:

(a) Applying FFT to the excitation signal and the direct wave signal to get $Y(0, \omega)$ and $Y(r, \omega)$

(b) Obtaining the wavenumber, $K(\omega)$, by adopting the phase spectrum measurement method in equations (11)-(13)

(c) Calculating the linear wavenumber, $K_{\operatorname{lin}}(\omega)$, and the wavenumber increment, $\Delta K(\omega)$, in equations (2) and (4) 


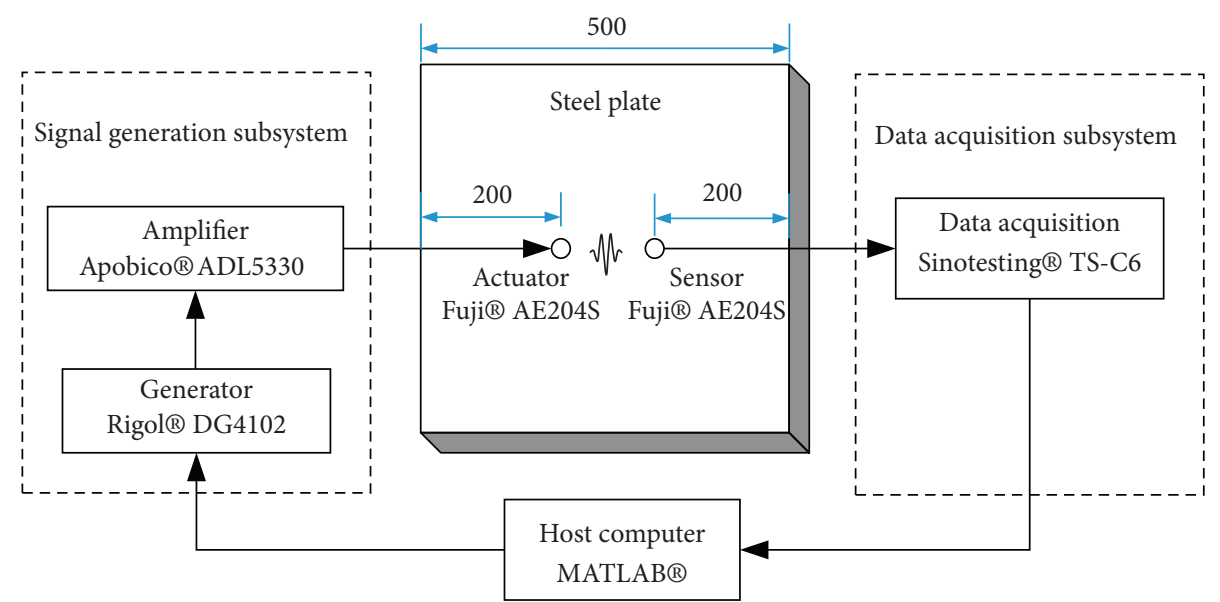

FIGURE 6: Sketch of the experiment setup with an actuator-sensor pair based on the Lamb wave.

(d) Performing the wavelet spectrum measurement method to estimate the flight time and propagating distance in equations (14)-(17)

(e) For each wavepacket, $Y_{\text {lin }}^{\prime}(r, t)$ is compensated by the phase-delay factor $e^{-i \Delta K(\omega) r}$ to $Y(r, \omega)$ with equation (9)

(f) For each wavepacket, correcting the waveform with $e^{-i \varphi_{0} r}$ to get $Y_{\text {lin }}(r, \omega)$

(g) Applying N-point IFFT to $Y_{\text {lin }}(r, f)$ to get $y_{\text {lin }}(r, t)$

3.4. Experiment Validation. To validate the $\triangle \mathrm{K}-\mathrm{DC}$ algorithm with the measured wavenumber, an experiment was conducted in an isotropic steel plate, with a size of $500 \times 500 \times 2 \mathrm{~mm}$. Two transducers (Fuji ${ }^{\circledR}$ AE204S) with a central frequency of $200 \mathrm{kHz}$ were perfectly surface-bonded on the plate using coupling gel, as indicated in Figure 6, where the left transducer is a wave actuator to produce Lamb waves and the right is a sensor. A 3.5-cycle sinusoid tone burst at a central frequency of $200 \mathrm{kHz}$ modulated by a Hanning window was generated by an arbitrary wave generator (Apobico ${ }^{\circledR}$ ADL5330). The excitation signals after being amplified to $50 \mathrm{~V}$ (peak to peak) (Rigol ${ }^{\circledR}$ DG4102) were applied to the actuator. The sensor signals were captured on the upper and lower surfaces upon traveling $100 \mathrm{~mm}$ by using a nonmetal ultrasonic detector (Sinotesting ${ }^{\circledR}$ TS-C6). The sampling frequency was $10 \mathrm{MHz}$. The NFFT point was set to 10,000 . The resolution was $1 \mathrm{kHz}$. The antisymmetric mode can be obtained by subtraction of the captured signals, as highlighted in Figure 7. There are two wavepackets: one for the direct $\mathrm{A}_{0}$ mode and another for the reflected $A_{0}$ wave from the boundary.

To begin the $\triangle \mathrm{K}$-DC process, the first step is to measure the wavenumber, $K(\omega)$, using the phase spectrum measurement method. Figures $8(\mathrm{a})$ and $8(\mathrm{~b})$ display the spectrum of the excitation signal and the direct $\mathrm{A}_{0}$ wave. After the phase is unwrapped, the phase difference spectrum, $\Delta \Phi(\omega)$, can be calculated based on equation (12), as shown in Figure 8(c). Then, the wavenumber is computed based on equation (13); the linear wavenumber is constructed based

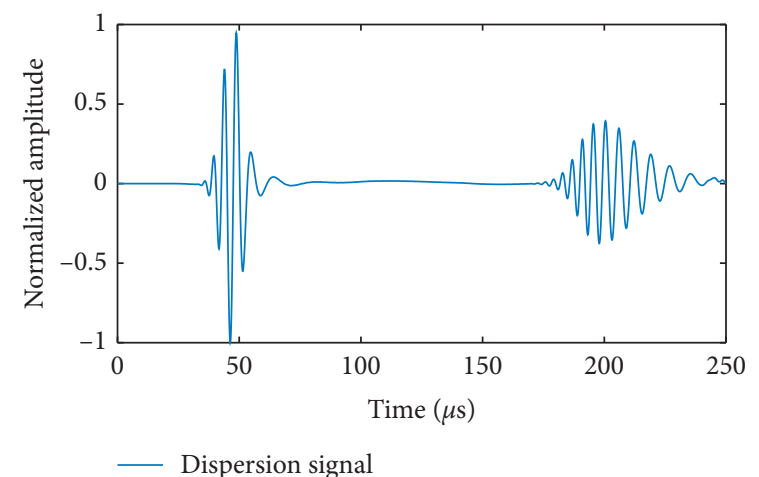

Figure 7: Raw signal with direct and reflected $\mathrm{A}_{0}$ waves, $y(r, t)$.

on equation (2); the delta wavenumber is obtained based on equation (4); Figure $8(\mathrm{~d})$ presents the above wavenumber relation. At frequency $200 \mathrm{kHz}$, the measured group velocity was $2647.9 \mathrm{~m} / \mathrm{s}$ vs. theoretical value of $2794.4 \mathrm{~m} / \mathrm{s}$ and the measured phase velocity was $1895.7 \mathrm{~m} / \mathrm{s}$ vs. theoretical value of $1724.8 \mathrm{~m} / \mathrm{s}$.

The wavelet energy spectrum method was used to estimate the traveling distance of each wavepacket. Looking at Figure 8(e), it is apparent that the arrival time of the direct $\mathrm{A}_{0}$ mode and the reflected $\mathrm{A}_{0}$ mode is the 465th and 1981st sampling points. And the arrival time of the excitation signal was the 88th sampling point (shown in Figure 4(c)). Based on equations (14)-(16), the estimated distances of the direct $\mathrm{A}_{0}$ mode and the reflected $\mathrm{A}_{0}$ mode were $99.8 \mathrm{~mm}$ and $501.2 \mathrm{~mm}$, which were in good agreement with the experimental expectations, $100 \mathrm{~mm}$ and $500 \mathrm{~mm}$.

Then, phase compensation was executed for the direct $A_{0}$ wave with $99.8 \mathrm{~mm}$ and reflected $\mathrm{A}_{0}$ wave with $501.2 \mathrm{~mm}$. Phase compensation is illustrated in Figures 8(f) and 8(g). It can be seen that the waveforms were compressed in the time domain but still different from the excitation. Lastly, phase compensation was applied. From Figure 8(h), we can see that, under the circumstances of multiple-wavepacket arrival, the $\triangle \mathrm{K}-\mathrm{DC}$ method can get good compensation to separately compensate each wavepacket using different traveling distances. 


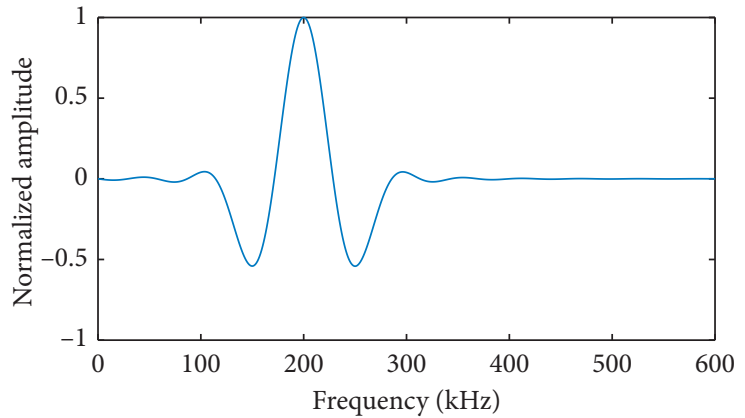

—_ Excitation signal

(a)

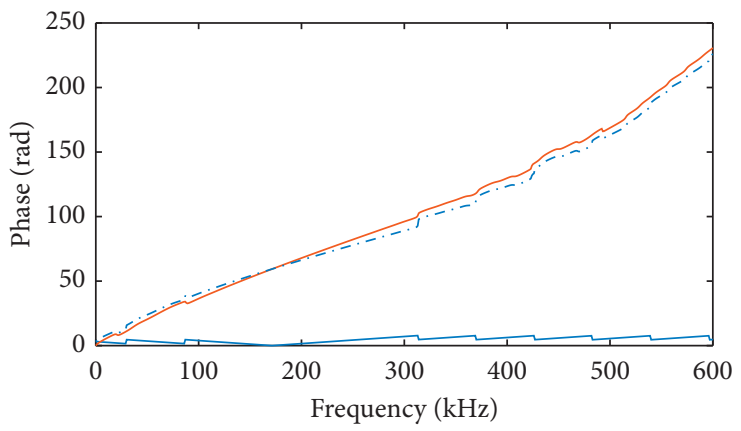

$-\Phi(y(0, t))$
$-\Phi(y(r, t))$
$--\Delta \phi$

(c)

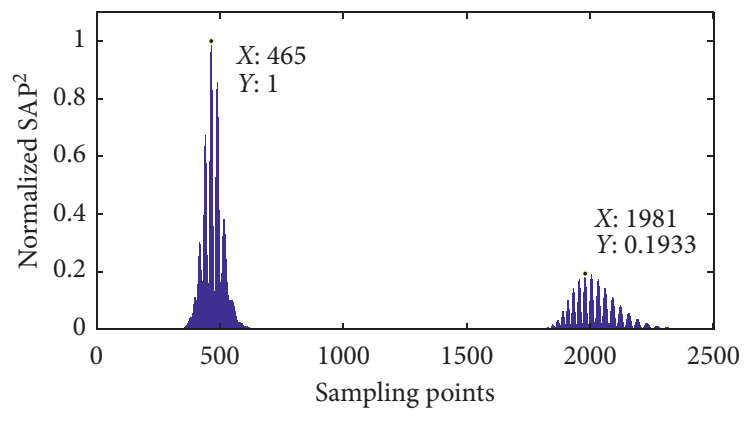

(e)

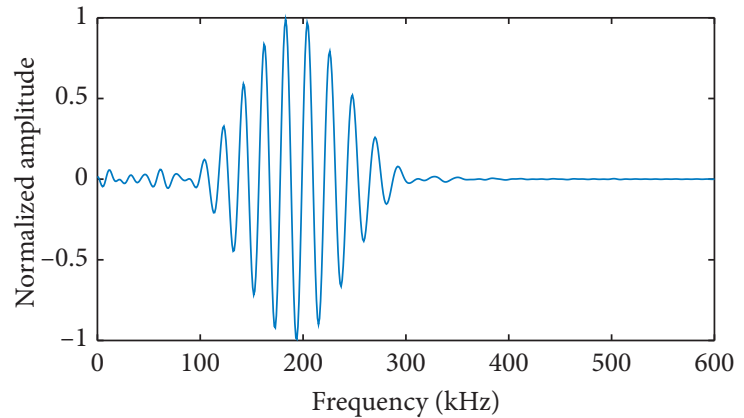

Direct $A_{0}$ wave

(b)

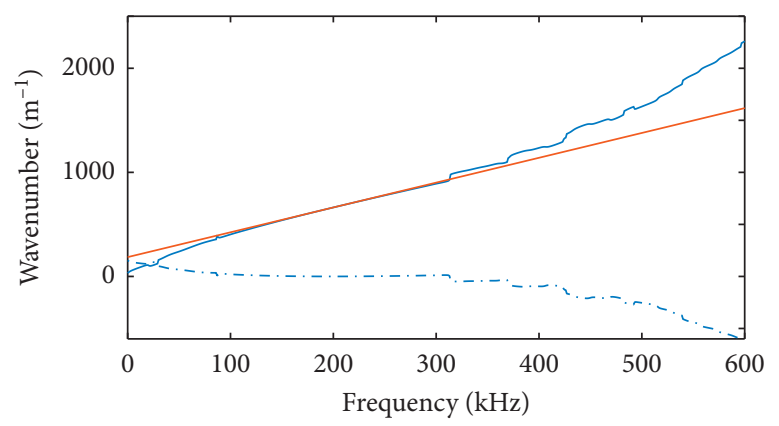

$-k=K(\omega)$

$-k_{\text {lin }}=K_{\text {lin }}(\omega)$

- - $\left.\Delta K=k_{\text {lin }}-k(\omega)\right)$

(d)

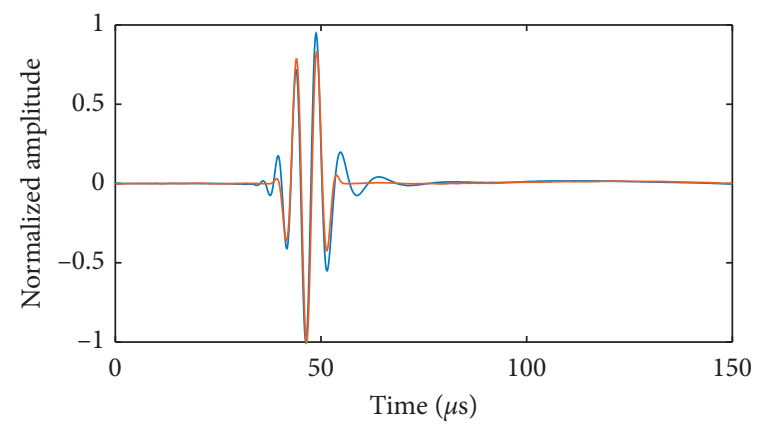

Direct $\mathrm{A}_{0}$ wave

Before correction

(f)

Figure 8: Continued. 


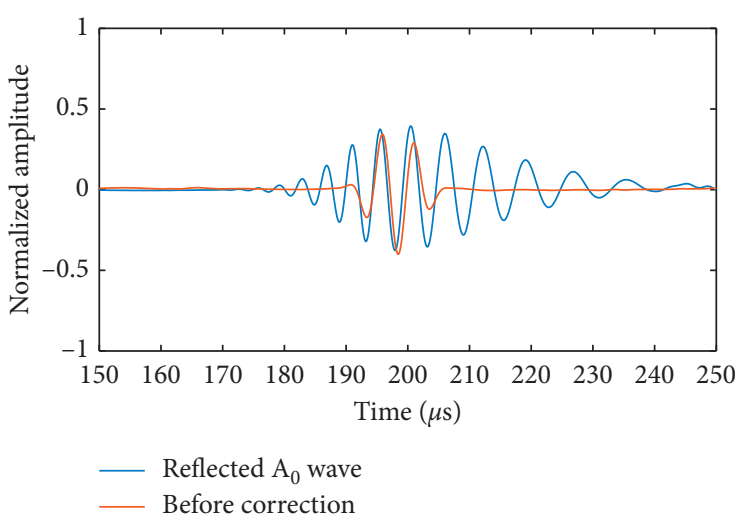

(g)

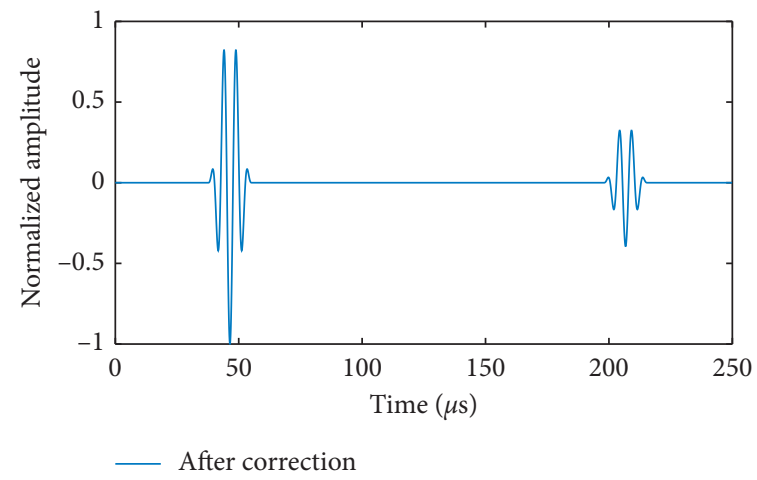

(h)

Figure 8: Procedure of $\triangle \mathrm{K}-\mathrm{DC}$ with the unknown wavenumber: (a) spectrum of the excitation signal $Y(0, \omega)$; (b) spectrum of the direct $\mathrm{A}_{0}$ wave $Y_{\mathrm{A}_{0}}(r, \omega)$; (c) phase spectrums $\Phi(0, \omega), \Phi(r, \omega)$, an $d \Delta \Phi(\omega)$; (d) wavenumber curves $K(\omega), K_{\operatorname{lin}}(\omega)$, an $d \Delta K(\omega)$; (e) SAP ${ }^{2}$ of the raw signal in Figure 7; (f) phase compensation result for the direct $\mathrm{A}_{0}$ wave $Y_{\text {lin }}^{\prime}(r, t)$ (direct $\mathrm{A}_{0}$ wave); (g) phase compensation result for the reflected $\mathrm{A}_{0}$ wave $Y_{\text {lin }}^{\prime}(r, t)$ (reflected $\mathrm{A}_{0}$ wave); (h) $\Delta \mathrm{K}$-DC result after correction $Y_{\text {lin }}(r, t)$.

\section{Conclusion}

This project was undertaken to propose a new $\triangle \mathrm{K}-\mathrm{DC}$ method for dispersion compensation of Lamb waves, which is applicable for waveguides with known or unknown material parameters. The applicability of $\Delta \mathrm{K}-\mathrm{DC}$ with the theoretical wavenumber and measured wavenumber was validated with a high-dispersive $\mathrm{A}_{0}$ mode in a $2 \mathrm{~mm}$ steel plate experiment. The results showed that $\triangle \mathrm{K}-\mathrm{DC}$ can get good compensation.

With regard to the $\triangle \mathrm{K}-\mathrm{DC}$ method, some limitations need to be acknowledged. Firstly, the propagation distance should be accurately known to measure the wavenumber. Secondly, when multiple modes propagate in a waveguide, it is necessary to identify the mode, measure the dispersion curve, and then apply $\triangle \mathrm{K}-\mathrm{DC}$ to each mode separately. Further investigation and experimentation of $\triangle \mathrm{K}-\mathrm{DC}$ into anisotropy composite media are strongly recommended for developing NDT techniques, such as damage location, damage quantification, and high resolution of damage imaging.

\section{Data Availability}

The data used to support the findings of this study areavailable from the corresponding author upon request.

\section{Conflicts of Interest}

The authors declare that they have no conflicts of interest.

\section{Acknowledgments}

Our heartfelt thanks are due to Prof. Han and Dr. Qin for their academic supervision and personal support. This work was supported by the Emergency Management Project of Natural Science Foundation of China (Grant no. 61842103), Youth Science Foundation (Grant no. 11604304), and Natural Science Foundation of Shanxi, China (Grant no. 201801D121156).

\section{References}

[1] P. D. Wilcox, R. P. Dalton, M. J. S. Lowe, and P. Cawley, "Mode and transducer selection for long range lamb wave inspection," Key Engineering Materials, vol. 167-168, pp. 152-161, 1999.

[2] M. Miniaci, A. S. Gliozzi, B. Morvan, A. Krushynska, and N. M. Pugno, "Proof of concept for an ultrasensitive technique to detect and localize sources of elastic nonlinearity using phononic crystals," Physical Review Letters, vol. 118, no. 21, Article ID 214301, 2017.

[3] T. Ghosh, T. Kundu, and P. Karpur, "Efficient use of Lamb modes for detecting defects in large plates," Ultrasonics, vol. 36, no. 7, pp. 791-801, 1998.

[4] S. U. Zhongqing, Identification of Damage Using Lamb Waves, Springer, Berlin, Germany, 2009.

[5] W. Ostachowicz, Guided Waves in Structures for SHM: The Time-domain Spectral Element Method, John Wiley \& Sons, Hoboken, NJ, USA, 2012.

[6] X. Hao, C. Li, S. Zhongqing, and G. Jean-Louis, "Identification of structural damage based on locally perturbed dynamic equilibrium with an application to beam component," Journal of Sound and Vibration, vol. 330, no. 24, pp. 5963-5981, 2011.

[7] J. Li and Y. Han, "Effects of adhesive parameters on dispersion characteristics of ultrasonic guided waves in composite pipes," Advances in Materials Science and Engineering, vol. 2019, Article ID 2735328, 10 pages, 2019.

[8] J. Cai, L. Shi, and X. P. Qing, "A time-distance domain transform method for Lamb wave dispersion compensation considering signal waveform correction," Smart Materials and Structures, vol. 22, no. 10, Article ID 105024, 2013.

[9] P. D. Wilcox, "A rapid signal processing technique to remove the effect of dispersion from guided wave signals," IEEE Transactions on Ultrasonics, Ferroelectrics and Frequency Control, vol. 50, no. 4, pp. 419-427, 2003.

[10] J. Cai, S. Yuan, X. P. Qing, F.-K. Chang, L. Shi, and L. Qiu, "Linearly dispersive signal construction of Lamb waves with measured relative wavenumber curves," Sensors and Actuators A: Physical, vol. 221, pp. 41-52, 2015.

[11] S. Fu, L. Shi, Y. Zhou, and J. Cai, "Dispersion compensation in lamb wave defect detection with step-pulse excitation and warped frequency transform," IEEE Transactions on 
Ultrasonics, Ferroelectrics, and Frequency Control, vol. 61, no. 12, pp. 2075-2088, 2014.

[12] R. K. Ing and M. Fink, "Time-reversed lamb waves," IEEE Transactions on Ultrasonics, Ferroelectrics and Frequency Control, vol. 45, no. 4, pp. 1032-1043, 1998.

[13] R. Gangadharan, C. R. L. Murthy, S. Gopalakrishnan, and M. R. Bhat, "Time reversal technique for health monitoring of metallic structure using Lamb waves," Ultrasonics, vol. 49, no. 8, pp. 696-705, 2009.

[14] F. Li, G. Meng, L. Ye, Y. Lu, and K. Kageyama, "Dispersion analysis of Lamb waves and damage detection for aluminum structures using ridge in the time-scale domain," Measurement Science and Technology, vol. 20, no. 9, Article ID 095704, 2009.

[15] M. Miniaci, M. Mazzotti, M. Radzienski et al., "Application of a laser-based time reversal algorithm for impact localization in a stiffened aluminium plate," Frontiers in Materials, vol. 6, 2019.

[16] S. C. Fu, Z. J. Lv, D. Ma, and L. H. Shi, "A method of dispersion compensation based on warped frequency transform," Advanced Materials Research, vol. 718-720, pp. 2062-2067, 2013.

[17] L. De Marchi, A. Marzani, and M. Miniaci, "A dispersion compensation procedure to extend pulse-echo defects location to irregular waveguides," NDT \& E International, vol. 54, pp. 115-122, 2013.

[18] L. De Marchi, A. Perelliand, and A. Marzani, "A signal processing approach to exploit chirp excitation in Lamb wave defect detection and localization procedures," Mechanical Systems \& Signal Processing, vol. 39, no. 1-2, pp. 20-31, 2013.

[19] R. Sicard, J. Goyette, and D. Zellouf, "A numerical dispersion compensation technique for time recompression of Lamb wave signals," Ultrasonics, vol. 40, no. 1-8, pp. 727-732, 2002.

[20] J. Hua, J. Lin, and L. Zeng, "Pulse compression and dispersion compensation for high- resolution Lamb wave inspection," Journal of Physics: Conference Series, vol. 628, Article ID 012122, 2015.

[21] C.-b. Xu, Z.-b. Yang, X.-f. Chen, S.-h. Tian, and Y. Xie, “A guided wave dispersion compensation method based on compressed sensing," Mechanical Systems and Signal Processing, vol. 103, pp. 89-104, 2018.

[22] L. J. Rose, Ultrasonic Guided Waves in Solid Media: Guided Wave Mode and Frequency Selection Tips, Cambridge University Press, Cambridge, UK, 2014.

[23] M. K. Hinders, J. Hou, and K. R. Leonard, "Automatic multimode Lamb wave arrival time extraction for improved tomographic reconstruction," Inverse Problems, vol. 20, 2005. 